PROCEEDINGS OF THE

AMERICAN MATHEMATICAL SOCIETY

Volume 130, Number 5, Pages 1425-1435

S 0002-9939(01)06228-1

Article electronically published on December 27, 2001

\title{
ULTRASTABILITY OF IDEALS OF HOMOGENEOUS POLYNOMIALS AND MULTILINEAR MAPPINGS ON BANACH SPACES
}

\author{
KLAUS FLORET AND STEPHAN HUNFELD
}

(Communicated by Dale Alspach)

\begin{abstract}
Using the theory of full and symmetric tensor norms on normed spaces, a theorem of Kürsten and Heinrich on ultrastability and maximality of normed operator ideals is extended to ideals of $n$-homogeneous polynomials and $n$-linear mappings - scalar-valued and vector-valued. The motivation for these results is the following important special case: the "uniterated" AronBerner extension $\bar{q}^{\mathfrak{U}}: E^{\prime \prime} \longrightarrow F^{\prime \prime}$ of an $n$-homogeneous polynomial $q: E \longrightarrow$ $F$ to the bidual remains in certain ideals under preservation of the norm. Moreover, Lotz's characterization of maximal normed ideals of linear mappings through appropriate tensor norms is proved for ideals of $n$-homogeneous scalarvalued polynomials and ideals of $n$-linear mappings.
\end{abstract}

\section{Preliminaries}

1.1. For vector spaces $E_{1}, \ldots, E_{n}, E, F$ over $\mathbb{K}=\mathbb{R}$ or $\mathbb{C}$ the "full" $n$-fold tensor product of $\left(E_{1}, \ldots, E_{n}\right)$ will be denoted by $E_{1} \otimes \cdots \otimes E_{n}=\otimes\left(E_{1}, \ldots, E_{n}\right)=$ $\otimes_{j=1}^{n} E_{j}$. The universal definition of the $n$-fold tensor product identifies the $n$-linear mappings $E_{1} \times \cdots \times E_{n} \longrightarrow F$ with the linear mappings $\otimes_{j=1}^{n} E_{j} \longrightarrow F$ :

$$
L\left(E_{1}, \ldots, E_{n} ; F\right)=L\left(\otimes_{j=1}^{n} E_{j} ; F\right), \quad \varphi \rightsquigarrow \varphi^{L} .
$$

Symmetric linear mappings $E^{n} \longrightarrow F$ are linearized by the $n$-th symmetric tensor product $\otimes^{n, s} E$ :

$$
L_{s}\left({ }^{n} E ; F\right)=L\left(\otimes^{n, s} E ; F\right), \quad \varphi \rightsquigarrow \varphi^{L, s}
$$

(see e.g. [F1] for more details). A mapping $q: E \longrightarrow F$ is, by definition, an $n$ homogeneous polynomial (notation: $q \in P^{n}(E ; F)$ ) if there is a $\varphi \in L\left({ }^{n} E ; F\right)$ with $q(x)=\varphi(x, \ldots, x)$ for all $x \in E$. Actually there is a unique $\check{q} \in L_{s}\left({ }^{n} E ; F\right)$ with this property:

$$
P^{n}(E ; F)=L_{s}\left({ }^{n} E ; F\right)=L\left(\otimes^{n, s} E ; F\right), \quad q \rightsquigarrow \check{q} \rightsquigarrow q^{L} .
$$

If the spaces are normed, then the continuous $n$-linear mappings and $n$-homogeneous polynomials are denoted by $\mathcal{L}\left(E_{1}, \ldots, E_{n} ; F\right), \mathcal{L}_{s}\left({ }^{n} E ; F\right)$ or $\mathcal{P}^{n}(E ; F)$, respectively.

Received by the editors February 9, 1999 and, in revised form, November 20, 2000.

2000 Mathematics Subject Classification. Primary 46B08; Secondary 46B28, 46G25.

Key words and phrases. Tensor products, symmetric tensor products, ideals of polynomials, ideals of $n$-linear mappings, ultraproducts. 
1.2. Again for fixed $n \in \mathbb{N}$ the projective norm $\pi$ is uniquely defined by the property

$$
\left[\otimes_{\pi}\left(E_{1}, \ldots, E_{n}\right)\right]^{\prime} \stackrel{1}{=} \mathcal{L}\left(E_{1}, \ldots, E_{n}\right): \stackrel{1}{=} \mathcal{L}\left(E_{1}, \ldots, E_{n} ; \mathbb{K}\right)
$$

if $E_{1}, \ldots, E_{n}$ are normed spaces ( $\stackrel{1}{=}$ means isometrically equal).

The injective norm $\varepsilon$ satisfies, by definition $(\stackrel{1}{\hookrightarrow}$ stands for a metric injection),

$$
\otimes_{\varepsilon}\left(E_{1}, \ldots, E_{n}\right) \stackrel{1}{\hookrightarrow}\left(\otimes_{\pi}\left(E_{1}^{\prime}, \ldots, E_{n}^{\prime}\right)\right)^{\prime} ;
$$

this shows that $\varepsilon$ is somehow dual to $\pi$. A tensor norm $\beta$ of order $n$ assigns to each $n$-tuple $\left(E_{1}, \ldots, E_{n}\right)$ a norm $\beta\left(\cdot ; E_{1}, \ldots, E_{n}\right)$ on $\otimes\left(E_{1}, \ldots, E_{n}\right)$ (notation: $\otimes_{\beta}\left(E_{1}, \ldots, E_{n}\right)$ or $\left.\otimes_{\beta, j=1}^{n} E_{j}\right)$ such that

(a) $\varepsilon \leq \beta \leq \pi$,

(b) $\left\|\otimes_{j=1}^{n} T_{j}: \otimes_{\beta, j=1}^{n} E_{j} \longrightarrow \otimes_{\beta, j=1}^{n} F_{j}\right\| \leq \prod_{j=1}^{n}\left\|T_{j}: E_{j} \longrightarrow F_{j}\right\|$ for all operators $T_{j} \in \mathcal{L}\left(E_{j} ; F_{j}\right)$ (the metric mapping property).

Note that (a) and (b) imply that in (b) there is actually equality. $\beta$ is called finitely generated if for all $E_{j}$ and $z \in \otimes_{j=1}^{n} E_{j}$

$$
\beta\left(z ; E_{1}, \ldots, E_{n}\right)=\inf \left\{\beta\left(z ; M_{1}, \ldots, M_{n}\right) \mid M_{j} \in \operatorname{FIN}\left(E_{j}\right), z \in \otimes_{j=1}^{n} M_{j}\right\}
$$

(where $\mathrm{FIN}(E)$ denotes the set of finite-dimensional subspaces of $E$ ). $\varepsilon$ and $\pi$ are finitely generated tensor norms of order $n$. There is no general reference for the theory of tensor norms of order $n>2$; many results, however, are straightforward generalizations of the case $n=2$ which, e.g., is treated in $\mathrm{DF}$.

1.3. In the same spirit the natural projective and injective norms $\pi_{s}$ and $\varepsilon_{s}$ on the $n$-th symmetric tensor product satisfy

$$
\begin{aligned}
\left(\otimes_{\pi_{s}}^{n, s} E\right)^{\prime} & \stackrel{1}{=} \mathcal{P}^{n}(E ; \mathbb{K})=: \mathcal{P}^{n}(E), \\
\otimes_{\varepsilon_{s}}^{n, s} E & \stackrel{1}{\hookrightarrow} \mathcal{P}^{n}\left(E^{\prime}\right) .
\end{aligned}
$$

An $s$-tensor norm $\alpha$ of order $n$ (or shortly $s$-tensor norm, if $n \in \mathbb{N}$ is clear) assigns to each normed space $E$ a norm $\alpha\left(\cdot ; \otimes^{n, s} E\right.$ ) on $\otimes^{n, s} E$ (notation: $\otimes_{\alpha}^{n, s} E$ ) such that

(a) $\varepsilon_{s} \leq \alpha \leq \pi_{s}$,

(b) the metric mapping property $\left\|\otimes^{n, s} T: \otimes_{\alpha}^{n, s} E \longrightarrow \otimes_{\alpha}^{n, s} F\right\| \leq\|T: E \longrightarrow F\|^{n}$ for all $T \in \mathcal{L}(E ; F)$.

$\alpha$ is called finitely generated if for all $E$ and $z \in \otimes^{n, s} E$,

$$
\alpha\left(z ; \otimes^{n, s} E\right)=\inf \left\{\alpha\left(z ; \otimes^{n, s} M\right) \mid M \in \operatorname{FIN}(E), z \in \otimes^{n, s} M\right\} .
$$

A detailed study of $\varepsilon_{s}$ and $\pi_{s}$ can be found in [F1]; the theory of $s$-tensor norms (in the spirit of Grothendieck's theory of tensor norms of order 2, see $[\overline{\mathrm{DF}}]$ ) will be developed in a forthcoming paper [F2]. We do not need anything from the general theory in this paper. Note that for convenience the definitions allow $n$ to be 1: in this case $\otimes_{\alpha}^{1} E \stackrel{1}{=} E \stackrel{1}{=} \otimes_{\alpha}^{1, s} E$.

1.4. Let $\mathfrak{U}$ be an ultrafilter on a set $I$; the ultraproduct (along $\mathfrak{U}$ ) of a family $\left(E_{\iota}\right)_{\iota \in I}$ of Banach spaces $E_{\iota}$ will be denoted by $\left(E_{\iota}\right)_{\mathfrak{U}}$ (see e.g. [DF, 18.4]). Now take $\varphi_{\iota} \in \mathcal{L}\left(E_{1, \iota}, \ldots, E_{n, \iota} ; F_{\iota}\right)$ for all $\iota \in I$ such that $\sup _{i \in I}\left\|\varphi_{\iota}\right\|<\infty$. Then

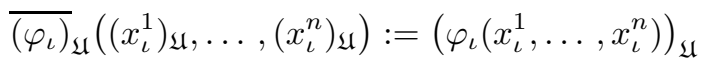


defines an $n$-linear map $\left(E_{1, \iota}\right)_{\mathfrak{U}} \times \cdots \times\left(E_{n, \iota}\right)_{\mathfrak{U}} \longrightarrow\left(F_{\iota}\right)_{\mathfrak{U}}$ between the ultraproducts; it easily follows that $\left\|{\overline{\left(\varphi_{\iota}\right)}}_{\mathfrak{U}}\right\|=\lim _{\mathfrak{U}}\left\|\varphi_{\iota}\right\|$. If $F_{\iota}=\mathbb{K}^{m}$, then

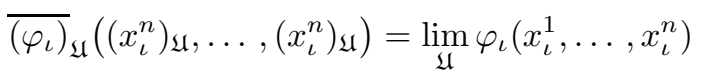

since $\left(\mathbb{K}^{m}\right)_{\mathfrak{U}}=\mathbb{K}^{m} ;$ in this case we write $\lim _{\mathfrak{U}} \varphi_{\iota}$ for $\overline{\left(\varphi_{\iota}\right)_{\mathfrak{U}}}$. If $E_{1, \iota}=\cdots=E_{n, \iota}=$ $E_{\iota}$, it is clear that $\left(\varphi_{\iota}\right)_{\mathfrak{U}}$ is symmetric if all $\varphi_{\iota}$ are; in particular, for polynomials $q_{\iota} \in \mathcal{P}^{n}\left(E_{\iota} ; F_{\iota}\right)$ with $\sup _{\iota}\left\|q_{\iota}\right\|<\infty$,

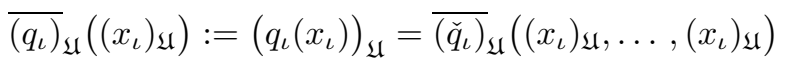

is an $n$-homogeneous polynomial $\left(E_{\iota}\right)_{\mathfrak{U}} \longrightarrow\left(F_{\iota}\right)_{\mathfrak{U}}$. Again it is rather immediate to see that

$$
\left(\mathcal{P}^{n}\left(E_{\iota} ; F_{\iota}\right)\right)_{\mathfrak{U}} \longrightarrow \mathcal{P}^{n}\left(\left(E_{\iota}\right)_{\mathfrak{U}} ;\left(F_{\iota}\right)_{\mathfrak{U}}\right), \quad\left(q_{\iota}\right)_{\mathfrak{U}} \longrightarrow{\overline{\left(q_{\iota}\right)_{\mathfrak{U}}}}
$$

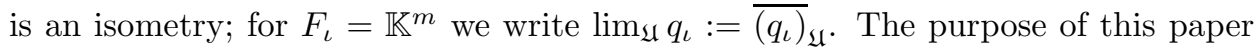
is to study under which circumstances $\overline{\left(q_{\iota}\right)_{\mathfrak{U}}}\left(\right.$ resp. $\overline{\left(\varphi_{\iota}\right)_{\mathfrak{U}}}$ ) is in a certain class of polynomials (resp. $n$-linear mappings) if all $q_{\iota}$ (resp. $\varphi_{\iota}$ ) are.

1.5. For this, two special properties of ultraproducts will be needed: local determination and local duality.

Proposition (local determination of ultraproducts). Let $E_{\iota}$ be normed spaces for $\iota \in I$ and $\{0\} \neq M \in \operatorname{FIN}\left(\left(E_{\iota}\right)_{\mathfrak{U}}\right)$. Then there are, for all $\iota \in I$, operators $R_{\iota} \in \mathcal{L}\left(M ; E_{\iota}\right)$ such that

(a) $x=\left(R_{\iota} x\right)_{\mathfrak{U}}$ for all $x \in M$;

(b) $\left\|R_{\iota}\right\| \leq 1$ for all $\iota \in I$ and there is an $\mathcal{U} \in \mathfrak{U}$ with $\left\|R_{\iota}\right\|=1$ for all $\iota \in \mathcal{U}$;

(c) for all $\varepsilon>0$ there is an $\mathcal{U}_{\varepsilon} \in \mathfrak{U}$ such that the inverse $R_{\iota}^{-1}: R_{\iota}(M) \longrightarrow M$ exists and $\left\|R_{\iota}^{-1}\right\| \leq 1+\varepsilon$ for all $\iota \in \mathcal{U}_{\varepsilon}$.

This is due to Kürsten [K, Satz 4.1] and Heinrich [H, Prop. 6.1]. Here we shall only need (a) and the first part of (b).

1.6. For the local duality, take normed spaces $E_{\iota}$ and denote by

$$
J:\left(E_{\iota}^{\prime}\right)_{\mathfrak{U}} \longrightarrow\left(E_{\iota}\right)_{\mathfrak{U}}^{\prime}, \quad J\left(x_{\iota}^{\prime}\right)_{\mathfrak{U}}=\lim _{\mathfrak{U}} x_{\iota}^{\prime}
$$

the natural map from 1.4., i.e., $\left\langle J\left(x_{\iota}^{\prime}\right)_{\mathfrak{U}},\left(x_{\iota}\right)_{\mathfrak{U}}\right\rangle=\lim _{\mathfrak{U}}\left\langle x_{\iota}^{\prime}, x_{\iota}\right\rangle$, and by

$$
K:\left(E_{\iota}\right)_{\mathfrak{U}} \hookrightarrow\left(E_{\iota}\right)_{\mathfrak{U}}^{\prime \prime} \stackrel{J^{\prime}}{\longrightarrow}\left(E_{\iota}^{\prime}\right)_{\mathfrak{U}}^{\prime} .
$$

Proposition (local duality of ultraproducts). Let $E_{\iota}$ be normed spaces for all $\iota \in$ $I, N \in \operatorname{FIN}\left(\left(E_{\iota}\right)_{\mathfrak{U}}^{\prime}\right)$ and $L \in \operatorname{FIN}\left(\left(E_{\iota}\right)_{\mathfrak{U}}\right)$. Then for every $\varepsilon>0$ there is an operator $T \in \mathcal{L}\left(N ;\left(E_{\iota}^{\prime}\right)_{\mathfrak{U}}\right)$ such that

(a) $\|T\|=1$ and $\left\|T^{-1}: T N \longrightarrow N\right\| \leq 1+\varepsilon$,

(b) $J T x^{\prime}=x^{\prime}$ for all $x^{\prime} \in N \cap \operatorname{im} J$,

(c) $\left\langle J T x^{\prime}, x\right\rangle=\left\langle K x, T x^{\prime}\right\rangle=\left\langle x^{\prime}, x\right\rangle$ for all $x^{\prime} \in N$ and $x \in L$.

This result is due to Kürsten [K], Stern (see $[\mathbf{H}]$ for references) and Heinrich [H]; the present formulation is taken from the proof of [ $\mathrm{H}$, Theorem 7.3]. We shall only need $\|T\|=1$ and (c). 


\section{THE MAIN THEOREM ON ULTRASTABILITY}

2.1. Every $z^{\prime} \in\left[\otimes_{k=1}^{m}\left(\otimes^{n_{k}, s} E_{k}\right)\right]^{*}$ defines a $\left(\sum_{k=1}^{m} n_{k}\right)$-linear functional $\varphi$ on $\prod_{k=1}^{m}\left(E_{k}\right)^{n_{k}}$ which is symmetric in the $n_{k}$ variables in $E_{k}$ for each $k=1, \ldots, m$, and vice-versa. In the spirit of the notations of 1.1 we may define $\varphi^{L}:=z^{\prime}$. For ultraproducts and $z_{\iota}^{\prime}=\varphi_{\iota}^{L}$ the notation

$$
\lim _{\mathfrak{U}} z_{\iota}^{\prime}:=\left[\lim _{\mathfrak{U}} \varphi_{\iota}\right]^{L}
$$

will be used in the following:

Theorem. Let $m, n_{1}, \ldots, n_{m} \in \mathbb{N}, \mathfrak{U}$ an ultrafilter on $I$, normed spaces $E_{k, \iota}, a$ finitely generated tensor norm $\beta$ of order $m$ and finitely generated s-tensor norms $\alpha_{k}$ of order $n_{k}$ be given. If $z_{\iota}^{\prime} \in\left[\otimes_{\beta, k=1}^{m}\left(\otimes_{\alpha_{k}}^{n_{k}, s} E_{k, \iota}\right)\right]^{\prime}=: H_{\iota}$ for all $\iota \in I$ such that $\sup _{\iota \in I}\left\|z_{\iota}^{\prime}\right\|_{H_{\iota}}<\infty$, then

$$
\lim _{\mathfrak{U}} z_{\iota}^{\prime} \in\left[\otimes_{\beta, k=1}^{m}\left(\otimes_{\alpha_{k}}^{n_{k}, s}\left(E_{k, \iota}\right)_{\mathfrak{U}}\right)\right]^{\prime}=: H
$$

and $\left\|\lim _{\mathfrak{U}} z_{\iota}^{\prime}\right\|_{H} \leq \lim _{\mathfrak{U}}\left\|z_{\iota}^{\prime}\right\|_{H_{\iota}}$.

Proof. Since $\beta$ and all $\alpha_{k}$ are finitely generated, it is enough to show that for all $M_{k} \in \operatorname{FIN}\left(\left(E_{k, \iota}\right) \mathfrak{U}\right)$ and all $z \in \otimes_{k=1}^{m}\left[\otimes^{n_{k}, s} M_{k}\right]=: M$,

$$
\left|\left\langle\lim _{\mathfrak{U}} z_{\iota}^{\prime}, z\right\rangle\right| \leq \lim _{\mathfrak{U}}\left\|z_{\iota}^{\prime}\right\|_{H_{\iota}} \cdot \beta\left(z ; \otimes_{\alpha_{1}}^{n_{1}, s} M_{1}, \ldots, \otimes_{\alpha_{m}}^{n_{m}, s} M_{m}\right)
$$

holds. Given these $M_{k}$ the local determination of ultraproducts (see 1.5) gives operators $R_{k, \iota}: M_{k} \longrightarrow E_{k, \iota}$ with $\left\|R_{k, \iota}\right\| \leq 1$ and $\left(R_{k, \iota} x_{k}\right)_{\mathfrak{U}}=x_{k}$ for all $x_{k} \in M_{k}$; it follows that

$$
\begin{aligned}
&\langle\lim _{\mathfrak{U}} z_{\iota}^{\prime}, \underbrace{\left[\otimes^{n_{1}} x_{1}\right] \otimes \cdots \otimes\left[\otimes^{n_{m}} x_{m}\right]}_{=: x}\rangle \\
&=\left\langle\lim _{\mathfrak{U}} z_{\iota}^{\prime},\left[\otimes^{n_{1}}\left(R_{1, \iota} x_{1}\right)_{\mathfrak{U}}\right] \otimes \cdots \otimes\left[\otimes^{n_{m}}\left(R_{m, \iota} x_{m}\right) \mathfrak{U}\right]\right\rangle \\
&=\lim _{\mathfrak{U}}\langle z_{\iota}^{\prime}, \underbrace{\left[\otimes^{n_{1}, s} R_{1, \iota}\right] \otimes \cdots \otimes\left[\otimes^{n_{m}, s} R_{m, \iota}\right]}_{=: R_{\iota}} x\rangle
\end{aligned}
$$

and hence for all $z \in M$,

$$
\begin{aligned}
\left|\left\langle\lim _{\mathfrak{U}} z_{\iota}^{\prime}, z\right\rangle\right| & =\left|\lim _{\mathfrak{U}}\left\langle z_{\iota}^{\prime}, R_{\iota} z\right\rangle\right| \\
& \leq\left|\lim _{\mathfrak{U}}\left\|z_{\iota}^{\prime}\right\|_{H_{\iota}} \beta\left(R_{\iota} z ; \otimes_{\alpha_{1}}^{n_{1}, s} E_{1, \iota}, \ldots, \otimes_{\alpha_{m}}^{n_{m}, s} E_{m, \iota}\right)\right| \\
& \leq\left|\lim _{\mathfrak{U}}\left\|z_{\iota}^{\prime}\right\|_{H_{\iota}}\left\|R_{1, \iota}\right\|^{n_{1}} \ldots\left\|R_{m, \iota}\right\|^{n_{m}} \beta\left(z ; \otimes_{\alpha_{1}}^{n_{1}} M_{1}, \ldots, \otimes_{\alpha_{m}}^{n_{m}} M_{m}\right)\right|
\end{aligned}
$$

by the metric mapping properties of $\beta$ and $\alpha_{1}, \ldots, \alpha_{m}$. This is the desired inequality.

In other words, the natural map

$$
\left(\left[\otimes_{\beta, k=1}^{m}\left(\otimes_{\alpha_{k}}^{n_{k}, s} E_{k, \iota}\right)\right]^{\prime}\right)_{\mathfrak{U}} \longrightarrow\left[\otimes_{\beta, k=1}^{m}\left(\otimes_{\alpha_{k}}^{n_{k}, s}\left(E_{k, L}\right)_{\mathfrak{U}}\right)\right]^{\prime}
$$

has norm $\leq 1$. It is rather immediate that the norm is 1 if all $E_{\iota} \neq\{0\}$ and that for $\beta=\pi$ and $\alpha_{k}=\pi_{s}$ this is even an isometry (as in the special cases of 1.4, one has to take $x_{k, \iota} \in B_{E_{k, \iota}}$ with $\left.\left|\left\langle x_{\iota}^{\prime}, \ldots\right\rangle\right| \geq\left\|x_{\iota}^{\prime}\right\|(1-\varepsilon)\right)$. 
2.2. Note the special cases of $m=1$ (polynomials) and all $n_{k}=1$ (no symmetry):

(a) If $\alpha$ is a finitely generated $s$-tensor norm of order $n$, then for all normed spaces $E_{\iota}$ the natural map

$$
\left(\left(\otimes_{\alpha}^{n, s} E_{\iota}\right)^{\prime}\right)_{\mathfrak{U}} \longrightarrow\left(\otimes_{\alpha}^{n, s}\left(E_{\iota}\right)_{\mathfrak{U}}\right)^{\prime}
$$

has norm $\leq 1$.

(b) If $\beta$ is a finitely generated tensor norm of order $m$, then for all normed spaces $E_{k, \iota}$ the natural map

$$
\left(\left(\otimes_{\beta}\left(E_{1, \iota}, \ldots, E_{m, \iota}\right)\right)^{\prime}\right)_{\mathfrak{U}} \longrightarrow\left(\otimes_{\beta}\left(\left(E_{1, \iota}\right)_{\mathfrak{U}}, \ldots,\left(E_{m, \iota}\right) \mathfrak{U}\right)\right)^{\prime}
$$

has norm $\leq 1$.

2.3. It is clear that the same reasoning gives that the natural map

$$
\left(\left[\otimes_{\alpha}^{m, s}\left(\otimes_{\beta, k=1}^{n} E_{k, \iota}\right)\right]^{\prime}\right)_{\mathfrak{U}} \longrightarrow\left[\otimes_{\alpha}^{m, s}\left(\otimes_{\beta, k=1}^{n}\left(E_{k, \iota}\right)_{\mathfrak{U}}\right)\right]^{\prime}
$$

has norm $\leq 1$.

\section{SCALAR-VALUED IDEALS OF POLYNOMIALS AND MULTILINEAR MAPPINGS}

3.1. A subclass $\mathcal{Q} \subset \mathcal{P}^{n}$ of $n$-homogeneous continuous scalar-valued polynomials on Banach spaces is called an ideal, if

(a) $\mathcal{Q}(E):=\mathcal{P}^{n}(E) \cap \mathcal{Q}$ is a linear subspace of $\mathcal{P}^{n}(E)$ for all Banach spaces $E$,

(b) if $T \in \mathcal{L}(E ; F)$ and $q \in \mathcal{Q}(F)$, then $q \circ T \in \mathcal{Q}$,

(c) $\left[\mathbb{K} \ni z \rightsquigarrow z^{n} \in \mathbb{K}\right] \in \mathcal{Q}$.

If $\|\cdot\|_{\mathcal{Q}}: \mathcal{Q} \longrightarrow[0, \infty]$ satisfies

$\left.\left(\mathrm{a}^{\prime}\right)\|\cdot\|_{\mathcal{Q}}\right|_{\mathcal{Q}(E)}$ is a norm for all Banach spaces $E$,

$\left(\mathrm{b}^{\prime}\right)\|q \circ T\|_{\mathcal{Q}} \leq\|T\|^{n}\|q\|_{\mathcal{Q}}$ in the situation of $(\mathrm{b})$,

$\left(\mathrm{c}^{\prime}\right)\left\|\mathbb{K} \ni z \rightsquigarrow z^{n} \in \mathbb{K}\right\|_{\mathcal{Q}}=1$,

then $\left(\mathcal{Q},\|\cdot\|_{\mathcal{Q}}\right)$ is called a normed ideal of n-homogeneous polynomials. It can easily be seen that always $\|q\| \leq\|q\|_{\mathcal{Q}}$ and that $\mathcal{Q}(M)=\mathcal{P}^{n}(M)=\otimes^{n, s} M^{\prime}$ for all finite-dimensional $M$. It would also be possible to define ideals of polynomials on normed spaces (not only Banach spaces) - but this would not make much of a difference.

It is rather immediate to see that for each $s$-tensor norm $\alpha$ of order $n$

$$
\mathcal{Q}(E):=\left(\otimes_{\alpha}^{n, s} E\right)^{\prime}
$$

defines a normed ideal of $n$-homogeneous polynomials. For $\alpha=\varepsilon_{s}$ one obtains the integral polynomials (see e.g. [F1, chap. 3] for their properties). It is not difficult to see that all extendible $n$-homogeneous polynomials (i.e., those $q \in \mathcal{P}^{n}(E)$ such that for all super spaces $G \supset E$ there is an extension $\widetilde{q} \in \mathcal{P}^{n}(G)$ of $G$; see Kirwan and Ryan $[\mathrm{KR}]$ ) are also of this form; in this case the $s$-tensor norm $\alpha$ of order $n$ is the "injective associate" of the projective $s$-norm $\pi_{s}$, i.e., satisfies

$$
\otimes_{\alpha}^{n, s} E \stackrel{1}{\hookrightarrow} \otimes_{\pi_{s}}^{n, s} \ell_{\infty}\left(B_{E^{\prime}}\right) ;
$$

we omit the details. 
3.2. For $\left(\mathcal{Q},\|\|_{\mathcal{Q}}\right)$ and $q \in \mathcal{P}^{n}(E)$ define

$$
\|q\|_{\mathcal{Q}_{\max }}:=\sup \left\{\left\|\left.q\right|_{M}\right\|_{\mathcal{Q}} \mid M \in \operatorname{FIN}(E)\right\} \in[0, \infty] .
$$

$\left(\mathcal{Q},\|\|_{\mathcal{Q}}\right)$ is called maximal if every $q \in \mathcal{P}^{n}(E)$ with $\|q\|_{\mathcal{Q}_{\max }}<\infty$ is in $\mathcal{Q}$ and $\|q\|_{\mathcal{Q}}=\|q\|_{\mathcal{Q}_{\max }}$. $\left(\mathcal{Q},\|\|_{\mathcal{Q}}\right)$ is called ultrastable if for $q_{\iota} \in \mathcal{Q}\left(E_{\iota}\right)$ with $\sup _{i \in I}\left\|q_{\iota}\right\|_{\mathcal{Q}}<\infty$ one has $\lim _{\mathfrak{U}} q_{\iota} \in \mathcal{Q}\left(\left(E_{\iota}\right)_{\mathfrak{U}}\right)$ and $\left\|\lim _{\mathfrak{U}} q_{\iota}\right\|_{\mathcal{Q}} \leq \sup \left\|q_{\iota}\right\|_{\mathcal{Q}}$ (and hence $\left.\leq \lim _{\mathfrak{U}}\left\|q_{\iota}\right\|_{\mathcal{Q}}\right)$.

Theorem. For each normed ideal $\left(\mathcal{Q},\|\|_{\mathcal{Q}}\right)$ of n-homogeneous (scalar-valued) polynomials the following statements are equivalent:

(1) $\left(\mathcal{Q},\|\|_{\mathcal{Q}}\right)$ is maximal.

(2) $\left(\mathcal{Q},\|\|_{\mathcal{Q}}\right)$ is ultrastable.

(3) There is a finitely generated s-tensor norm $\alpha$ of order $n$ such that

$$
\mathcal{Q}(E) \stackrel{1}{=}\left(\otimes_{\alpha}^{n, s} E\right)^{\prime}
$$

for all Banach spaces E.

It is clear that this result can be conjectured when knowing the Kürsten-Heinrich characterization of maximal Banach operator ideals [H. Theorem 8.1] and Lotz' representation with tensor norms of order 2 (see [DF, 17.5]. It follows from (3) that $\mathcal{Q}(E)$ is a Banach space if $\mathcal{Q}$ is maximal.

Proof. (1) $\curvearrowright$ (3). For finite-dimensional Banach spaces $M$ define $\alpha$ by

$$
\otimes_{\alpha}^{n, s} M:=\mathcal{Q}(M)^{\prime}
$$

and for arbitrary normed spaces $E$ by

$$
\alpha\left(z ; \otimes^{n, s} E\right):=\inf \left\{\alpha\left(z ; \otimes^{n, s} M\right) \mid M \in \operatorname{FIN}(E), z \in \otimes^{n, s} M\right\} .
$$

It is straightforward to see that $\alpha$ is a finitely generated $s$-tensor norm of order $n$ with $(*) \mathcal{Q}(M) \stackrel{1}{=}\left(\otimes_{\alpha}^{n, s} M\right)^{\prime}$ if $M$ is finite-dimensional. Now

$$
\begin{aligned}
\left\|q^{L}\right\|_{\left(\otimes_{\alpha}^{n, s} E\right)^{\prime}} & =\sup \left\{\left|\left\langle q^{L}, z\right\rangle\right| \mid \alpha\left(z ; \otimes^{n, s} E\right)<1\right\} \\
& =\sup \left\{\left|\left\langle\left(\left.q\right|_{M}\right)^{L}, z\right\rangle\right| \mid M \in \operatorname{FIN}(E), z \in \otimes^{n, s} M, \alpha\left(z ; \otimes^{n, s} M\right)<1\right\} \\
& \stackrel{(*)}{=} \sup \left\{\left\|\left.q\right|_{M}\right\|_{\mathcal{Q}} \mid M \in \operatorname{FIN}(E)\right\}=\|q\|_{\mathcal{Q}}
\end{aligned}
$$

by the maximality of $\mathcal{Q}$.

$(3) \curvearrowright(2)$. This is the special case 2.2(a) of the main theorem.

$(2) \curvearrowright(1)$. Let $q \in \mathcal{P}^{n}(E)$ with $\|q\|_{\mathcal{Q}_{\max }}<\infty$ and let $\mathfrak{U}$ be an ultrafilter finer than the order filter in $\operatorname{FIN}(E)$. For $\left.q\right|_{M}$ on $M \in \operatorname{FIN}(E)$ one obtains $\left\|\left.q\right|_{M}\right\|_{\mathcal{Q}} \leq\|q\|_{\mathcal{Q}_{\max }}$ and $\left.\lim _{\mathfrak{U}} q\right|_{M}:(M)_{\mathfrak{U}} \longrightarrow \mathbb{K}$ extends $q$ (via the natural isometric embedding $E \hookrightarrow$ $\left.(M)_{\mathfrak{U}}\right)$. If follows from $(2)$ that $q \in \mathcal{Q}$ and

$$
\|q\|_{\mathcal{Q}} \leq\left\|\left.\lim _{\mathfrak{U}} q\right|_{M}\right\|_{\mathcal{Q}} \leq \sup \left\|\left.q\right|_{M}\right\|_{\mathcal{Q}}=\|q\|_{\mathcal{Q}_{\max }}
$$

and hence $\|q\|_{\mathcal{Q}}=\sup \left\|\left.q\right|_{M}\right\|_{\mathcal{Q}}$ since always $\|\cdot\|_{\mathcal{Q}_{\max }} \leq\|\cdot\|_{\mathcal{Q}}$. 
3.3. Every $q \in \mathcal{P}^{n}(E)$ has an extension to $\mathcal{P}^{n}\left(E^{\prime \prime}\right)$ : the Aron-Berner extension (see $\mathrm{AB}$ ) can easily be seen as an iterated limit along local ultrafilters of $E$ (see [LR] or [F1, 6.9]). This motivated Dineen and Timoney [DT] and Lindström and Ryan LR independently to define an "uniterated" Aron-Berner extension (as it is called in [F1]) as follows: for $\left.\left.\iota:=(M, N, \varepsilon) \in I:=\mathrm{FIN}\left(E^{\prime \prime}\right) \times \mathrm{FIN}\left(E^{\prime}\right) \times\right] 0,1\right]$ choose with the strong principle of local reflexity an operator $T_{\iota} \in \mathcal{L}(M ; E)$ with $T_{\iota} x=x$ for all $x \in M \cap E$ such that $\left\|T_{\iota}\right\| \leq 1+\varepsilon$ and $\left\langle T_{\iota} x^{\prime \prime}, x^{\prime}\right\rangle$ for all $\left(x^{\prime \prime}, x^{\prime}\right) \in M \times N$; for $x^{\prime \prime} \in E^{\prime \prime}$ define $f_{\iota}\left(x^{\prime \prime}\right):=T_{\iota} x^{\prime \prime}$ if $x^{\prime \prime} \in M$ and $f_{\iota}\left(x^{\prime \prime}\right):=0$ otherwise. Take a local ultrafilter $\mathfrak{U}$ on $I$, i.e., an ultrafilter which is finer than the order filter on $I$, then the natural mappings

$$
\begin{aligned}
& J_{E}: E^{\prime \prime} \quad \longrightarrow(E)_{\mathfrak{U}} ; \quad x^{\prime \prime} \quad \rightsquigarrow\left(f_{\iota}\left(x^{\prime \prime}\right)\right)_{\mathfrak{U}}, \\
& Q_{E}:(E)_{\mathfrak{U}} \longrightarrow E^{\prime \prime} ; \quad\left(x_{\iota}\right)_{\mathfrak{U}} \rightsquigarrow \lim _{\iota, \mathfrak{U}} x_{\iota}
\end{aligned}
$$

$\left(\sigma\left(E^{\prime \prime}, E^{\prime}\right)\right.$-limit) have the following properties: $J_{E}$ is an isometry which extends the natural embedding $E \hookrightarrow(E)_{\mathfrak{U}}$, the mapping $Q_{E}$ has norm 1 (if $E \neq\{0\}$ ) and $J_{E} Q_{E}:(E)_{\mathfrak{U}} \longrightarrow \operatorname{im} J_{E} \stackrel{1}{=} E^{\prime \prime}$ is a norm-1-projection. For $q \in \mathcal{P}^{n}(E)$ define

$$
\bar{q}^{\mathfrak{U}}:=\left[\lim _{\iota, \mathfrak{U}} q\right] \circ J_{E} \in \mathcal{P}^{n}\left(E^{\prime \prime}\right)
$$

hence $\bar{q}^{\mathfrak{U}}\left(x^{\prime \prime}\right)=\lim _{\iota, \mathfrak{U}} q\left(f_{\iota}\left(x^{\prime \prime}\right)\right)$; it is clear that $\bar{q}^{\mathfrak{U}}$ extends $q$. The foregoing theorem implies the following:

Theorem. Let $\mathcal{Q}$ be a maximal normed ideal of n-homogeneous polynomials, $q \in$ $\mathcal{P}^{n}(E)$ and $\mathfrak{U}$ a local ultrafilter of $E$. Then $q \in \mathcal{Q}(E)$ if and only if $\bar{q}^{\mathfrak{U}} \in \mathcal{Q}\left(E^{\prime \prime}\right)$; in this case $\left\|\bar{q}^{\mathfrak{U}}\right\|_{\mathcal{Q}}=\|q\|_{\mathcal{Q}}$.

In particular, this applies to the class of integral polynomials. Note that it took considerable effort to prove this result for the iterated Aron-Berner extension in the cases $\mathcal{Q}:=\mathcal{P}^{n}$ (Davie-Gamelin $[\overline{\mathrm{DG}}]$ ) and $\mathcal{Q}:=\{$ integral polynomials $\}$ (due to [CZ]; in [F1, 6.8] there is an alternative proof).

3.4. It is clear that a theorem like 3.1 holds also for normed ideals of $n$-linear functionals originally defined by Pietsch $[\mathrm{P}]$ in 1983: a subclass $\mathcal{A}$ of all $n$-linear continuous functionals on Banach spaces is an ideal if (for all Banach spaces $E_{j}$ and $F_{j}$ )

(a) $\mathcal{A}\left(E_{1}, \ldots, E_{n}\right):=\mathcal{A} \cap \mathcal{L}\left(E_{1}, \ldots, E_{n}\right)$ is a linear subspace of $\mathcal{L}\left(E_{1}, \ldots, E_{n}\right)$,

(b) if $T_{j} \in \mathcal{L}\left(E_{j} ; F_{j}\right)$ and $\varphi \in \mathcal{A}\left(E_{1}, \ldots, E_{n}\right)$, then $\varphi \circ\left(T_{1}, \ldots, T_{n}\right) \in \mathcal{A}$,

(c) $\left[\mathbb{K}^{n} \ni\left(x_{1}, \ldots, x_{n}\right) \rightsquigarrow x_{1} \cdots x_{n} \in \mathbb{K}\right] \in \mathcal{A}$.

If $\|\cdot\|_{\mathcal{A}}: \mathcal{A} \longrightarrow[0, \infty]$ satisfies

$\left.\left(\mathrm{a}^{\prime}\right)\|\cdot\|_{\mathcal{A}}\right|_{\mathcal{A}\left(E_{1}, \ldots, E_{n}\right)}$ is a norm on $\mathcal{A}\left(E_{1}, \ldots, E_{n}\right)$,

$\left(\mathrm{b}^{\prime}\right)\left\|\varphi \circ\left(T_{1}, \ldots, T_{n}\right)\right\|_{\mathcal{A}} \leq\|T\| \cdots\left\|T_{n}\right\|\|\varphi\|_{\mathcal{A}}$ in the situation of $(\mathrm{b})$,

$\left(\mathrm{c}^{\prime}\right)\left\|\mathbb{K}^{n} \ni\left(x_{1}, \ldots, x_{n}\right) \rightsquigarrow x_{1} \cdots x_{n} \in \mathbb{K}\right\|_{\mathcal{A}}=1$,

then $\left(\mathcal{A},\|\|_{\mathcal{A}}\right)$ is called a normed ideal of n-linear functionals. It is obvious from 3.2 how to define that $\left(\mathcal{A},\|\|_{\mathcal{A}}\right)$ is maximal or ultrastable. The same kind of ideas as in the proof of Theorem 3.2 gives the following:

Theorem. For every normed ideal $\left(\mathcal{A},\|\|_{\mathcal{A}}\right)$ of n-linear functionals the following statements are equivalent:

(1) $\left(\mathcal{A},\|\|_{\mathcal{A}}\right)$ is maximal.

(2) $\left(\mathcal{A},\|\|_{\mathcal{A}}\right)$ is ultrastable. 
(3) There exists a finitely generated tensor norm $\beta$ of order $n$ with

$$
\left(\otimes_{\beta}\left(E_{1}, \ldots, E_{n}\right)\right)^{\prime} \stackrel{1}{=} \mathcal{A}\left(E_{1}, \ldots, E_{n}\right)
$$

for all Banach spaces $E_{1}, \ldots, E_{n}$.

\section{VECTOR-VALUED IDEALS OF POLYNOMIALS AND MULTILINEAR MAPPINGS}

4.1. A normed ideal of $n$-homogeneous continuous vector-valued polynomials on Banach spaces is, by definition, a pair $\left(\mathcal{Q},\|\|_{\mathcal{Q}}\right)$ such that:

(a) $\mathcal{Q}(E ; F):=\mathcal{Q} \cap \mathcal{P}^{n}(E ; F)$ is a linear subspace of $\mathcal{P}^{n}(E ; F)$ and $\left.\|\|_{\mathcal{Q}}\right|_{\mathcal{Q} E ; F)}$ is a norm on it.

(b) If $T \in \mathcal{L}\left(E_{1} ; E_{2}\right), q \in \mathcal{Q}\left(E_{2} ; E_{3}\right)$ and $S \in \mathcal{L}\left(E_{3} ; E_{4}\right)$, then $S \circ q \circ T \in \mathcal{Q}$ and $\|S \circ q \circ T\|_{\mathcal{Q}} \leq\|T\|^{n}\|q\|_{\mathcal{Q}}\|S\|$.

(c) $\left[\mathbb{K} \ni z \rightsquigarrow z^{n} \in \mathbb{K}\right]$ is in $\mathcal{Q}$ and has norm 1 .

4.2. We are only interested in ideals coming from tensor norms. For this the following notation will be used:

$$
\begin{aligned}
\mathcal{L}\left(E ; G^{\prime}\right) & =\left(E \otimes_{\pi} G\right)^{\prime}, \quad T \rightsquigarrow \beta_{T}, \\
\mathcal{P}^{n}\left(E ; G^{\prime}\right) & =\mathcal{L}\left(\otimes_{\pi_{s}}^{n, s} E ; G^{\prime}\right)=\left(\left(\otimes_{\pi_{s}}^{n, s} E\right) \otimes_{\pi} G\right)^{\prime}, \quad q \rightsquigarrow \beta_{q}:=\beta_{q^{L}} .
\end{aligned}
$$

Let $\alpha$ be a finitely generated $s$-tensor norm of order $n$ and $\beta$ a finitely generated tensor norm of order 2 . We define $q \in \mathcal{P}_{(\alpha, \beta)}^{n}(E ; F)$ if $\beta_{\left(\varkappa_{F} \circ q\right)} \in\left(\left(\otimes_{\alpha}^{n, s} E\right) \otimes_{\beta} F^{\prime}\right)^{\prime}$ and in this case $\|q\|_{(\alpha, \beta)}:=\left\|\beta_{\varkappa_{F} \circ q}\right\|_{(\ldots)}$ (here $\varkappa_{F}: F \hookrightarrow F^{\prime \prime}$ is the natural injection). It is easy to see that $\left(\mathcal{P}_{(\alpha, \beta)}^{n},\|\|_{(\alpha, \beta)}\right)$ is a normed ideal in the sense of 4.1. If $\mathcal{C}$ is the maximal Banach operator ideal associated with the dual tensor norm $\beta^{\prime}$ of $\beta$, then from the representation theorem [DF, 17.5] we get

$$
\mathcal{P}_{(\alpha, \beta)}^{n}(E ; F) \stackrel{1}{=} \mathcal{C}\left(\widetilde{\otimes}_{\alpha}^{n, s} E ; F\right) .
$$

A consequence of this is that $\mathcal{P}_{(\alpha, \beta)}^{n}$ is regular, i.e., $q \in \mathcal{P}_{(\alpha, \beta)}^{n}(E ; F)$ if (and only if) $\varkappa_{F} \circ q \in \mathcal{P}_{(\alpha, \beta)}^{n}\left(E ; F^{\prime \prime}\right)$ and $\|q\|_{(\alpha, \beta)}=\left\|\varkappa_{F} \circ q\right\|_{(\alpha, \beta)}$. It is also rather routine to show that $\mathcal{P}_{(\alpha, \beta)}^{n}$ is maximal in the following sense: $q \in \mathcal{P}^{n}(E ; F)$ is in $\mathcal{P}_{(\alpha, \beta)}^{n}$ if

$$
\sup \left\{\left\|\left.Q_{L}^{F} \circ q\right|_{M}\right\|_{(\alpha, \beta)} \mid L \in \operatorname{COFIN}(E), M \in \operatorname{FIN}(E)\right\}<\infty
$$

and then this number is $\|q\|_{(\alpha, \beta)}$ (where $\operatorname{COFIN}(E):=\{L \subset E / L$ finite-codimensional, closed subspace of $E)=\left\{N^{0} \mid N \in \mathrm{FIN}\left(E^{\prime}\right)\right\}$ and $Q_{L}^{F}: F \longrightarrow F / L$ the canonical quotient map). However, it seems to be unlikely that every maximal and $\operatorname{regular}\left(\mathcal{Q},\|\|_{\mathcal{Q}}\right)$ is of the form $\mathcal{P}_{(\alpha, \beta)}^{n}$, contrary to the scalar case.

Just one example: Since $\left[\otimes_{\varepsilon_{s}}^{n, s} E\right] \otimes_{\varepsilon} F \stackrel{1}{\hookrightarrow} \mathcal{P}^{n}\left(E^{\prime} ; F\right)$, one can deduce from [JM. Lemma 2.1.] (see also [A2] for the reflexive case) that $\mathcal{P}_{\varepsilon_{s}, \varepsilon}^{n}\left(E ; F^{\prime}\right)$ is the space of integral $n$-homogeneous polynomials $E \longrightarrow F^{\prime}$ in the sense of Alencar A1].

Theorem. The ideal $\mathcal{P}_{(\alpha, \beta)}^{n}$ is ultrastable, i.e., if $\mathfrak{U}$ is an ultrafilter on $I$ and $q_{\iota} \in$ $\mathcal{P}_{(\alpha, \beta)}^{n}\left(E_{\iota} ; F_{\iota}\right)$ such that $\sup \left\|q_{\iota}\right\|_{(\alpha, \beta)}<\infty$, then $\overline{\left(q_{\iota}\right)_{\mathfrak{U}}} \in \mathcal{P}_{(\alpha, \beta)}^{n}\left(\left(E_{\iota}\right)_{\mathfrak{U}} ;\left(F_{\iota}\right)_{\mathfrak{U}}\right)$ and

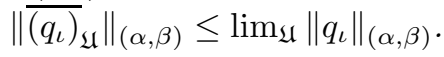

See 1.4 for the notation. 


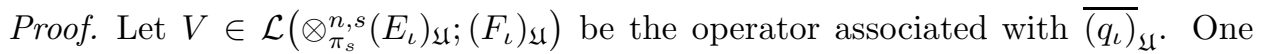
has to show that $\widetilde{V} \in \mathcal{C}\left(\widetilde{\otimes}_{\alpha}^{n, s}\left(E_{\iota}\right)_{\mathfrak{U}} ;\left(F_{\iota}\right)_{\mathfrak{U}}\right)$ where $(\mathcal{C}, \mathbf{C})$ is the Banach operator ideal associated with $\beta^{\prime}$ and $\widetilde{V}$ is the extension of $V$ to the completion $\widetilde{\otimes}_{\alpha}^{n, s}\left(E_{\iota}\right)_{\mathfrak{U}}$. From the main theorem in Section 2 we get

$$
z^{\prime}:=\lim _{\mathfrak{U}} \beta_{\varkappa_{F_{\iota}} \circ q_{\iota}} \in\left[\left(\otimes_{\alpha}^{n, s}\left(E_{\iota}\right)_{\mathfrak{U}}\right) \otimes_{\beta}\left(F_{\iota}^{\prime}\right)_{\mathfrak{U}}\right]^{\prime}=: H
$$

and $\left\|z^{\prime}\right\|_{H} \leq \lim _{\mathfrak{U}}\left\|q_{\iota}\right\|_{(\alpha, \beta)}$. If $z^{\prime}=\beta_{U}$ with $U \in \mathcal{L}\left(\widetilde{\otimes}_{\alpha}^{n, s}\left(E_{\iota}\right)_{\mathfrak{U}} ;\left(F_{\iota}^{\prime}\right)_{\mathfrak{U}}^{\prime}\right)$ (hence $\left.\mathbf{C}(U)=\left\|z^{\prime}\right\|_{H}\right)$, then $U=K \circ \widetilde{V}$ where $K:\left(F_{\iota}\right)_{\mathfrak{U}}=: F \longrightarrow\left(F_{\iota}^{\prime}\right)_{\mathfrak{U}}^{\prime}=: G^{\prime}$ is the natural mapping from 1.6 (check on $\left.\left[\otimes^{n}\left(x_{\iota}\right)_{\mathfrak{U}}\right] \otimes\left(x_{\iota}^{\prime}\right)_{\mathfrak{U}}\right)$. Using maximality it is enough to show that

$$
\left\|M \hookrightarrow \widetilde{\otimes}_{\alpha}^{n, s}\left(E_{\iota}\right)_{\mathfrak{U}} \stackrel{\widetilde{V}}{\longrightarrow} F \stackrel{Q_{N^{0}}^{F}}{\longrightarrow} F / N^{0}=N^{\prime}\right\| \leq\left\|z^{\prime}\right\|_{H}
$$

for all $M \in \operatorname{FIN}\left(\widetilde{\otimes}_{\alpha}^{n, s}\left(E_{\iota}\right)_{\mathfrak{U}}\right)$ and $N \in \operatorname{FIN}\left(F^{\prime}\right)$. With $L:=\widetilde{V}(M) \subset F$ the local duality 1.6 of ultraproducts gives an operator $T \in \mathcal{L}(N ; G)$ with $\|T\|=1$ and

$$
\left\langle K \widetilde{V}(u), T x^{\prime}\right\rangle_{G^{\prime}, G}=\left\langle x^{\prime}, \widetilde{V}(u)\right\rangle_{F^{\prime}, F}=\left\langle Q_{N^{0}}^{F} \widetilde{V}(u), x^{\prime}\right\rangle_{N^{\prime}, N}
$$

for all $u \in M$ and $x^{\prime} \in N$. This means $\left.Q_{N^{0}}^{F} \circ \tilde{V}\right|_{M}=\left.T^{\prime} \circ K \circ \widetilde{V}\right|_{M}$. It follows that $\mathbf{C}\left(\left.Q_{N^{0}}^{F} \circ \widetilde{V}\right|_{M}\right) \leq\left\|T^{\prime}\right\| \mathbf{C}\left(\left.K \circ \widetilde{V}\right|_{M}\right)=\mathbf{C}\left(\left.U\right|_{M}\right) \leq\left\|z^{\prime}\right\|_{H}$ and therefore $\left\|{\overline{\left(q_{\iota}\right)}}_{\mathfrak{U}}\right\|_{(\alpha, \beta)}=\mathbf{C}(\widetilde{V}) \leq \lim _{\mathfrak{U}}\left\|q_{\iota}\right\|_{(\alpha, \beta)}$.

4.3. Note the special case $\beta=\pi$ and $q_{\iota}^{0}: E_{\iota} \longrightarrow \otimes_{\alpha}^{n, s} E_{\iota}$ being the "canonical" polynomial $q_{\iota}^{0}(x):=\otimes^{n} x$, i.e., $\left(q_{\iota}^{0}\right)^{L}=\operatorname{id}_{\otimes_{\alpha}^{n, s} E_{\iota}}$ and $\left\|q_{\iota}^{0}\right\|_{(\alpha, \pi)}=1$. Then ${\overline{\left(q_{\iota}^{0}\right)_{\mathfrak{U}}}}_{\text {is }}$ in $\mathcal{P}_{\alpha, \pi}\left(\left(E_{\iota}\right)_{\mathfrak{U}} ;\left(\widetilde{\otimes}_{\alpha}^{n, s} E_{\iota}\right)_{\mathfrak{U}}\right)$ which means that the natural map

$$
\otimes_{\alpha}^{n, s}\left(E_{\iota}\right)_{\mathfrak{U}} \longrightarrow\left(\otimes_{\alpha}^{n, s} E_{\iota}\right)_{\mathfrak{U}}
$$

defined by $\otimes^{n}\left(x_{\iota}\right)_{\mathfrak{U}} \rightsquigarrow\left(\otimes^{n} x_{\iota}\right)_{\mathfrak{U}}$ has norm $\leq 1$, if $\alpha$ is a finitely generated $s$-tensor norm.

4.4. For any ultrafilter $\mathfrak{U}$ and normed space $F$ the map $Q_{F}:(F)_{\mathfrak{U}} \longrightarrow F^{\prime \prime}$ from 3.3 is well-defined, extends the natural embedding $F \hookrightarrow(F)_{\mathfrak{U}}$ and satisfies

$$
\left\langle Q_{F}\left(\left(y_{\iota}\right)_{\mathfrak{U}}\right), y^{\prime}\right\rangle_{F^{\prime \prime}, F^{\prime}}=\lim _{\iota, \mathfrak{U}}\left\langle y_{\iota}, y^{\prime}\right\rangle_{F, F^{\prime}}
$$

If $\mathfrak{U}$ is a local ultrafilter of another space $E$ and $q \in \mathcal{P}^{n}(E ; F)$, then (see 3.3 for the notation)

$$
\bar{q}^{\mathfrak{U}}:=Q_{F} \circ \overline{(q)}_{\mathfrak{U}} \circ J_{E}: E^{\prime \prime} \longrightarrow F^{\prime \prime}
$$

is an $n$-homogeneous polynomial which extends $q$ and can be calculated as follows:

$$
\left\langle\bar{q}^{\mathfrak{U}}\left(x^{\prime \prime}\right), y^{\prime}\right\rangle_{F^{\prime \prime}, f^{\prime}}=\lim _{\iota, \mathfrak{U}}\left\langle q\left(f_{\iota}\left(x^{\prime \prime}\right)\right), y^{\prime}\right\rangle .
$$

Theorem 4.2 and the regularity of $\mathcal{P}_{(\alpha, \beta)}^{n}$ imply the following:

Corollary. Take $q \in \mathcal{P}^{n}(E ; F)$ and a local ultrafilter on $E$. Then the extension $\bar{q}^{\mathfrak{U}}$ is in $\mathcal{P}_{(\alpha, \beta)}^{n}\left(E^{\prime \prime} ; F^{\prime \prime}\right)$ if and only if $q$ is; in this case $\|q\|_{(\alpha, \beta)}=\left\|\bar{q}^{\mathfrak{U}}\right\|_{(\alpha, \beta)}$. 
4.5. For $n$-linear operators $E_{1} \times \cdots \times E_{n} \longrightarrow F$ the same ideas apply: a normed ideal of n-linear continuous operators between Banach spaces is a pair $\left(\mathcal{A},\|\|_{\mathcal{A}}\right)$ such that

(a) $\mathcal{A}\left(E_{1}, \ldots, E_{n} ; F\right)=\mathcal{A} \cap \mathcal{L}\left(E_{1}, \ldots, E_{n} ; F\right)$ is linear and $\left.\|\|_{\mathcal{A}}\right|_{\mathcal{A}\left(E_{1}, \ldots, E_{n} ; F\right)}$ is a norm,

(b) if $T_{j} \in \mathcal{L}\left(G_{j} ; E_{j}\right), \varphi \in \mathcal{A}\left(E_{1}, \ldots, E_{n} ; F\right)$ and $S \in \mathcal{L}(F ; G)$, then the composition $S \circ \varphi \circ\left(T_{1}, \ldots, T_{n}\right)$ is in $\mathcal{A}$ and

$$
\left\|S \circ \varphi \circ\left(T_{1}, \ldots, T_{n}\right)\right\|_{\mathcal{A}} \leq\|S\|\|\varphi\|_{\mathcal{A}}\left\|T_{1}\right\| \cdots\left\|T_{n}\right\|
$$

(c) $\left[\mathbb{K}^{n} \ni\left(x_{1}, \ldots, x_{n}\right) \rightsquigarrow x_{1} \cdots x_{n} \in \mathbb{K}\right]$ is in $\mathcal{A}$ and $\|\cdots\|_{\mathcal{A}}=1$.

Every tensor norm $\beta$ of order $n+1$ defines an ideal $\mathcal{A}_{\beta}$ as follows: an $n$-linear map $\varphi$ is in $\mathcal{A}_{\beta}\left(E_{1}, \ldots, E_{n} ; F\right)$ if and only if the $(n+1)$-linear form associated with $\varkappa_{F} \circ \varphi$ is in $\left[\otimes_{\beta}\left(E_{1}, \ldots, E_{n}, F^{\prime}\right)\right]^{\prime}$.

An ideal $\left(\mathcal{A},\|\|_{\mathcal{A}}\right)$ is called maximal if

$$
\|\varphi\|_{\mathcal{A}^{\max }}:=\sup \left\{\left\|\left.Q_{L}^{F} \circ \varphi\right|_{M_{1} \times \cdots \times M_{n}}\right\|_{\mathcal{A}} \mid M_{j} \in \operatorname{FIN}\left(E_{j}\right), L \in \operatorname{COFIN}(F)\right\}<\infty
$$

implies $\varphi \in \mathcal{A}$ and $\|\varphi\|_{\mathcal{A}}=\|\varphi\|_{\mathcal{A}^{\max }}$ holds. The ideal is called regular if $\varkappa_{F} \circ \varphi \in$ $\mathcal{A}\left(E_{1}, \ldots, E_{n} ; F^{\prime \prime}\right)$ implies $\varphi \in \mathcal{A}$ and $\|\varphi\|_{\mathcal{A}}=\left\|\varkappa_{F} \circ \varphi\right\|_{\mathcal{A}}$; it is easy to see that the ideals $\mathcal{A}_{\beta}$ are regular. $\mathcal{A}$ is ultrastable if for $\varphi_{\iota} \in \mathcal{A}\left(E_{1, \iota}, \ldots, E_{n, \iota} ; F_{\iota}\right)$ with $\|\varphi\|_{\mathcal{A}} \leq c$ the operator $\overline{\left(\varphi_{\iota}\right)_{\mathfrak{U}}}$ is in $\mathcal{A}\left(\left(E_{1, \iota}\right)_{\mathfrak{U}}, \ldots,\left(E_{n, \iota}\right)_{\mathfrak{U}} ;\left(F_{\iota}\right)_{\mathfrak{U}}\right)$ and $\|{\overline{\left(\varphi_{\iota}\right)_{\mathfrak{U}}}}_{\|_{\mathcal{A}} \leq} \leq$ $\sup \left\|\varphi_{\iota}\right\|_{\mathcal{A}}$.

Theorem. Let $(\mathcal{A},\|\|)_{\mathcal{A}}$ be a normed ideal of $n$-linear continuous mappings between Banach spaces. Then the following statements are equivalent:

(1) $\left(\mathcal{A},\|\|_{\mathcal{A}}\right)$ is maximal.

(2) $\left(\mathcal{A},\|\|_{\mathcal{A}}\right)$ is ultrastable and regular.

(3) There is a finitely generated tensor norm $\beta$ of order $n+1$ such that

$$
\begin{aligned}
& \mathcal{A}\left(E_{1}, \ldots, E_{n} ; F^{\prime}\right) \stackrel{1}{=}\left(\otimes_{\beta}\left(E_{1}, \ldots, E_{n}, F\right)\right)^{\prime}, \\
& \mathcal{A}\left(E_{1}, \ldots, E_{n} ; F\right) \stackrel{1}{=}\left(\otimes_{\beta}\left(E_{1}, \ldots, E_{n}, F^{\prime}\right)\right)^{\prime} \cap \mathcal{L}\left(E_{1}, \ldots, E_{n} ; F\right) .
\end{aligned}
$$

Proof. (1) $\curvearrowright$ (3) runs exactly as in the case $n=1$ (see [DF 17.5.], the extension lemma holds also for finitely generated tensor norms of arbitrary order) with a construction of $\beta$ as in the proof of Theorem 3.2.

(3) $\curvearrowright(2)$. Ultrastability follows from the main theorem as in the proof of Theorem 4.2; the regularity follows immediately when looking at the $(n+1)$-linear functionals appearing in the two formulae in (3).

$(2) \curvearrowright(1)$. Take $\varphi \in \mathcal{L}\left(E_{1}, \ldots, E_{n} ; F\right)$ with $\|\varphi\|_{\mathcal{A}^{\max }}<\infty$. Following Heinrich's proof for the case $n=1$ (see [H]) consider $I:=\operatorname{FIN}\left(E_{1}\right) \times \cdots \times \operatorname{FIN}\left(E_{n}\right) \times \operatorname{FIN}\left(F^{\prime}\right)$ and let $\mathfrak{U}$ be an ultrafilter finer than the order filter. For $\iota=\left(M_{1}, \ldots, M_{n}, N\right)$ define $E_{k, \iota}:=M_{k}$ and $F_{\iota}:=F / N^{0}$ and metric embeddings

$$
J_{k}: E_{k} \longrightarrow\left(E_{k, \iota}\right)_{\mathfrak{U}} \quad x_{k} \rightsquigarrow\left(x_{k, \iota}\right)_{\mathfrak{U}}
$$

where $x_{k, \iota}=x_{k}$ if $x_{k} \in M_{k}$ and $=0$ otherwise. Moreover, define a mapping $Q:\left(F_{\iota}\right)_{\mathfrak{U}} \longrightarrow F^{\prime \prime}$ by

$$
\left\langle Q\left(y_{\iota}\right)_{\mathfrak{U}}, y^{\prime}\right\rangle:=\lim _{\mathfrak{U}}\left\langle y_{\iota}, y_{\iota}^{\prime}\right\rangle
$$

where $y_{\iota}^{\prime}=y^{\prime}$ if $y^{\prime} \in N$ and $=0$ otherwise; $Q$ has norm $\leq 1$.

For $\varphi_{\iota}:=\left.Q_{N^{0}}^{F} \circ \varphi\right|_{M_{1} \times \cdots \times M_{n}}$ one obtains

$$
\varkappa_{F} \circ \varphi=Q \circ{\overline{\left(\varphi_{\iota}\right)}}_{\mathfrak{U}} \circ\left(J_{1}, \ldots, J_{n}\right) \in \mathcal{A}
$$


with $\left\|\varkappa_{F} \circ \varphi\right\|_{\mathcal{A}} \leq \lim _{\iota}\|\varphi\|_{\mathcal{A}} \leq\|\varphi\|_{\mathcal{A}^{\max }}$; the regularity gives $\varphi \in \mathcal{A}$, and $\|\varphi\|_{\mathcal{A}}=$ $\left\|\varkappa_{F} \circ \varphi\right\|_{\mathcal{A}} \leq\|\varphi\|_{\mathcal{A}^{\max }}$.

For $n=1$ the equivalence $(1) \curvearrowleft(2)$ is the Heinrich-Kürsten result for Banach operator ideals and $(2) \curvearrowright(3)$ Lotz' respresentation theorem. Note that the result implies that all $\mathcal{A}\left(E_{1}, \ldots, E_{n} ; F\right)$ are Banach spaces if $\left(\mathcal{A},\|\|_{\mathcal{A}}\right)$ is maximal.

4.6. As in 4.3 one obtains that the natural map

$$
\otimes_{\gamma, k=1}^{n}\left(E_{k, \iota}\right)_{\mathfrak{U}} \longrightarrow\left(\otimes_{\gamma, k=1}^{n} E_{k, \iota}\right)_{\mathfrak{U}}
$$

defined by $\left(x_{\iota}^{\prime}\right)_{\mathfrak{U}} \otimes \cdots \otimes\left(x_{\iota}^{n}\right)_{\mathfrak{U}} \rightsquigarrow\left(x_{\iota}^{1} \otimes \cdots \otimes x_{\iota}^{n}\right)_{\mathfrak{U}}$ has norm $\leq 1$ if $\gamma$ is a finitely generated tensor norm or order $n$. For a proof define the tensor norm $\beta$ of order $n+1$ by

$$
\otimes_{\beta}\left(E_{1}, \ldots, E_{n}, E_{n+1}\right):=\left[\otimes_{\gamma}\left(E_{1}, \ldots, E_{n}\right)\right] \otimes_{\pi} E_{n+1},
$$

and apply the theorem to $\otimes: E_{1, \iota} \times \cdots \times E_{n, \iota} \longrightarrow \widetilde{\otimes}_{\gamma}\left(E_{1, \iota}, \ldots, E_{n, \iota}\right)=: F_{\iota}$.

\section{REFERENCES}

[A1] R. Alencar, On reflexivity and basis of $P\left({ }^{m} E\right)$, Proc. Roy. Irish Acad. 85 (1985), 131-138. MR 87i:46101

[A2] R. Alencar, An application of Singer's theorem to homogeneous polynomials, Contemp. Math. 144 (1993), 1-8. MR 94c:53087

[AB] R. Aron, P. Berner, A Hahn-Banach extension theorem for analytic mappings, Bull. Soc. Math. France 106 (1978), 3-24. MR 80e:46029

[CD] D. Carando, V. Dimant, Duality in spaces of nuclear and integral polynomials, J. Math. Anal. Appl. 241 (2000), 107-121. MR 2001c:46089

[CZ] D. Carando, I. Zalduendo, A Hahn-Banach theorem for integral polynomials, Proc. Amer. Math. Soc. 127 (1999), 241-250. MR 99b:46067

[DF] A. Defant, K. Floret, Tensor Norms and Operator Ideals, North Holland Math. Studies 176, 1993. MR 94e:46130

[DG] A. Davie, T. Gamelin, A theorem on polynomial-star approximation, Proc. Amer. Math. Soc. 106 (1989), 351-356. MR 89k:46023

[DT] S. Dineen, R.M. Timoney, Complex geodesics on convex domains, in: Progress Funct. Anal. (eds.: Bierstedt, Bonet, Horvath, Maestre) North Holland Math. Studies 170 (1992), 333365. MR 92m:46066

[F1] K. Floret, Natural norms on symmetric tensor products of normed spaces, Note di Matematica (Trier-conference 1997) 17 (1997), 153-188. MR 2001g:46038

[F2] K. Floret, The metric theory of symmetric tensor products of normed spaces, in preparation.

$[\mathrm{H}]$ S. Heinrich, Ultraproducts in Banach space theory, J. Reine Angew. Math. 313 (1980), 77-104. MR 82b:46013

[JM] J.A. Jaramillo, L.A. de Moraes, Duality and reflexivity in spaces of polynomials, Arch. Math. (Basel) 74 (2000), 282-293. MR 2000k:46063.

[K] K.D. Kürsten, s-Zahlen und Ultraprodukte von Operatoren in Banachräumen, Doctoral Thesis, Leipzig, 1976.

[KR] P. Kirwan, R. Ryan, Extendibility of homogeneous polynomials on Banach spaces, Proc. AMS 126 (1998), 1023-1029. MR 98f:46042

[LR] M. Lindström, R. Ryan, Applications of ultraproducts to infinite dimensional holomorphy, Math. Scand. 71 (1992), 229-242. MR 94c:46090

[P] A. Pietsch, Ideals of multilinear functionals, Proc. $2^{\text {nd }}$ Int. Conf. Operator Alg., etc. (Teubner Texte Math. 62), Leipzig (1984), 185-199. MR 85g:00027

Department of Mathematics, University of Oldenburg, D-26111 Oldenburg, Germany E-mail address: floret@mathematik.uni-oldenburg.de

Werstener Dorfstrasse 209, D-40591 Düsseldorf, Germany 